\title{
An Arts-Based Educational Exhibit on Menopausal Hot Flashes
}

Running title: Educational Hot Flash Exhibit

Janet S. Carpenter, PhD, RN, FAAN, School of Nursing, Indiana University, Indianapolis, Mark Kesling, BS, MS, The daVinci Pursuit, Indianapolis, Karen K. Schmidt, MSN, RN, School of Nursing, Indiana University, Indianapolis.

Funding: The work outlined in this paper was funded by: (1) an Indiana University sabbatical award (no number) for development of the exhibit concept; (2) an Indiana University Extraordinary Opportunities Travel Grant (no number) to develop portions of the exhibit concept); and (3) a Pfizer Inc. Independent Grant for Learning and Change (36068923) to build the exhibit prototype and research public feedback).

Conflicts: None.

Address for correspondence and reprints: Dr. Janet S. Carpenter, Indiana University School of Nursing, 600 Barnhill Drive, Room NU338, Indianapolis, IN, 46202. Phone: 317-278-6093, Fax: 317-278-2021, Email: carpentj@iu.edu.

Acknowledgements: We acknowledge additional members of our design team (Brittany Harvey, Chip Doeden, Bridget Gurtowsky of Gurtowsky Graphics, Rocky Walls and the group at 12 Stars Media, and J.K. and Megan Stewart at Paired Inc.).

This is the author's manuscript of the article published in final edited form as:

Carpenter, J. S., Kesling, M., \& Schmidt, K. K. (2019). An arts-based educational exhibit on menopausal hot flashes. Menopause (New York, N.Y.), 26(9), 1062-1067. 


\section{ABSTRACT}

Objective: To describe the development process, science, and symbolism of an arts-based educational exhibit designed to address myths, misinformation, negative imagery, and use of unproven treatments related to menopausal hot flashes.

Methods: The development process included iterative and informal feedback from a variety of individuals, a partnership with an experienced exhibit designer, and collaborations between artists and scientists.

Results: The resulting exhibit creates an environment where the public is immersed in accurate information about hot flashes. Although based on an iterative process, the resulting exhibit content reflects an estimated 500+ scientific studies, including those referenced in the NAMS position statements on hormone and non-hormone management of hot flashes. The seven main exhibit pieces convey scientific information and symbolize various aspects of women's experiences.

Conclusions: This innovative exhibit has high potential to be a disruptive innovation to address the preponderance of myths, misinformation, and negative imagery surrounding menopausal hot flashes and potentially decrease use of unproven therapies.

Keywords: women's health, menopausal hot flashes, arts-based science education, arts-based dissemination of science 


\section{INTRODUCTION}

Menopausal hot flashes (i.e., night sweats, vasomotor symptoms) are a global issue. These everyday symptoms will affect $75 \%$ of women, ${ }^{1}$ or about 21 million women living in the United States today and 1.1 billion women around the world by $2025 .^{1-5}$ The sudden rush of heat and sweating that defines hot flashes lasts only minutes but these symptoms typically persist for 7 years (range $=0$ to $15+$ years). ${ }^{1,6}$

There is a preponderance of myths, misinformation, and negative imagery surrounding menopausal hot flashes. Confusion about hot flashes is the norm. ${ }^{7-11}$ While many cultures use school health education classes as a forum for teaching young girls about the changes of adolescence and the onset of menses, no similar forum exists for teaching midlife women or others about the cessation of menses. Women are responsible for seeking information themselves and frequently encounter myths and misinformation. ${ }^{8,12,13}$ Available information can portray menopause as a loss or disease of hormone deficiencies and hot flashes as dreadful signs of aging, ${ }^{14-17}$ imaginary, taboo, and/or a joke. ${ }^{18,19}$ Studies show that many women (1) do not get the information they need and are confused about treatment options ${ }^{20-23}$ and (2) have partners who are also confused or do not know how to support them. ${ }^{11,24}$ In addition, use of unproven therapies at menopause/postmenopause is a pervasive issue..$^{25,26}$

To innovatively address these problems and create a new and more balanced collective image of menopausal hot flashes, we turned to the creative arts. The creative arts represent a unique way to challenge old truths, motivate dialogue and social action around an issue, and build a new collective, public image of a topic. ${ }^{27,28}$ The creative arts can convey a large amount of highly specialized and nuanced information in a succinct, compassionate, and understandable manner. ${ }^{28,}$

${ }^{29}$ The arts are capable of reaching highly diverse audiences, surpassing age, gender, 
socioeconomic, and other sociocultural boundaries as well as boundaries of artistic knowledge or

preference. ${ }^{30,31}$ By engaging multiple sensory channels, the creative arts generate an embodied experience and can promote learning and changes in behaviors, beliefs, and attitudes. ${ }^{28,32-36}$ Using the creative arts in this manner is called arts-based science education, arts-based knowledge translation, arts-based dissemination of science, and educational entertainment. Thus, it seemed appropriate to use the arts as the platform for addressing the problem.

Our arts-based exhibit is an educational resource for the public, which we define to include menopausal women, healthcare providers, and other men and women (teens to adults). The exhibit aims to convey accurate information, quell myths and misinformation, normalize menopause as a natural stage of reproductive aging, and possibly decrease use of unproven therapies. In this paper, we describe methods in the exhibit's development process and its resulting science and symbolism.

\section{METHODS}

\section{The Development process}

The exhibit concept stemmed from a series of sketches created by the first author as a way of organizing thoughts about the topics that could be included in an educational resource for the public. The sketches emerged spontaneously via the creative process and were not drawn systematically according to an a priori plan. It quickly became apparent that the resource could be artistic and visual. Literature searches revealed the value of the creative arts as described above.

As the exhibit concept took shape, the first author sought feedback iteratively and informally. Twelve people reacted to a verbal description of the exhibit idea and 10 reviewed sketches and a concept paper. The 22 people included 15 national leaders in women's health or arts-based 
dissemination of science, four female faculty colleagues, and the director and two staff members of an international menopause professional organization. These individuals provided feedback on the overall concept, scope, and breadth of the exhibit. Although there were some discussions about whether to include other menopausal symptoms, the consensus was that a focus on hot flashes was appropriate based on the amount of information to be conveyed and fact that an exhibit like this had not been previously attempted. Thus, additional information was not added to the exhibit from this feedback.

In the process of obtaining feedback, the first author started an informal partnership with the second author who is an experienced exhibit designer and leader of a non-profit organization connecting art, science, and the community. The second author assembled artists and an engineer to be part of an exhibit design team. The design team further vetted the concept and produced professional illustrations from the initial sketches.

Some of the professional sketches were included in applications to fund various portions of the work related to the exhibit. The teams conducting the funded work consist of scientists and artists. Internal funding has provided a means to develop some of the exhibit pieces, videodocument the exhibit work, and travel to show the exhibit. External funding provided a means to obtain focus group feedback to refine the exhibit and measures of its impact as well as hold public viewing events to obtain additional feedback on a prototype exhibit. The prototype exhibit consisted of 12 professionally designed graphic renderings of what the full-scale exhibit and exhibit pieces will look like, written explanations of the exhibit pieces (in English and Spanish), a professionally designed logo, and a 32-inch foam board model with miniaturized 3dimensional printed exhibit pieces. The data obtained in these funded projects will be published elsewhere and will be useful for identifying target audiences, securing exhibit display 
venues/locations, marketing, and designing research to evaluate the impact of the full-scale exhibit. To be able to build and show the full-scale exhibit locally, nationally, and beyond, there will be an ongoing need for funding.

\section{RESULTS}

Science and symbolism in the exhibit

The exhibit creates an environment where the public is immersed in accurate information about hot flashes, the cardinal symptom of menopause. ${ }^{1,16}$ We estimate that the resulting exhibit represents findings from over 500 scientific studies conducted by nurses, anthropologists, epidemiologists, physicians, psychologists, and others. Studies contained within the North American Menopause Society position statements on the hormone and non-hormone management of hot flashes ${ }^{9,10}$ are included in this estimate. References to support the science underlying each piece are too numerous to include here, however, in Table 1, we provide examples of representative information sources.

The overall exhibit is circular as a symbol of femininity and wholeness. There is an inner circle for a central exhibit space and an outer circle consisting of a storage area and entrance and exit hallways. There are crossbars for hanging exhibit pieces, lighting, and channeling electrical power. The central exhibit space does not have a clear path from one end to another. The intent is for attendees to wander through the central exhibit in symbolism of the meandering paths that many women take in their search to find information and treatments for bothersome symptoms. Inside the central exhibit space, there are seven main exhibit pieces (Table 1). Seven symbolizes one piece for each year that women experience hot flashes (on average). Six of the pieces contain 3-dimensional elements and one is a film. All pieces are based on scientific evidence and artistic symbolism. For example, "Hot Flashes in Different Cultures" (Table 1 and Figure 1) is based on 
evidence from epidemiological surveys and anthropological studies describing inter-individual variation in hot flashes. In particular, work by Dr. Lynette Leidy-Sievert has shown that Bangladeshi women experience hot flashes on the top of their heads whereas Mexican women experience hot flashes as a sticky sweat on the backs of their neck. ${ }^{37-39}$ Other women feel hot flashes from their chests up to their faces. This type of explanation is part of the written descriptions that accompany the exhibit art. We include this work as an example from the exhibit because to date, public feedback indicated this piece is a favorite. Because of its popularity, we used the conceptual graphic illustration (Figure 1) to build it full-scale (Figure 2).

The exhibit logo mimics the round exhibit shape (Figure 3). A woman's profile is embedded in the red hot flash flames. The blue edges are symbolic of cupped hands that are holding space for women. The edges are open to symbolize the flow of new information and new experiences happening inside the exhibit.

\section{CONCLUSIONS}

The exhibit itself is highly innovative. It represents an entirely different channel for meeting the needs of menopausal women. It is significantly different from a popular musical (> 11 million attendees worldwide $)^{40}$ that provides comedic relief rather than scientific information and has not been studied for its impact. The exhibit's format is also significantly different from decision aids and other interventions that have been shown to improve knowledge, ${ }^{41-43}$ ability to make informed and values-based decisions, ${ }^{43}$ and quality of life $^{41}$ at the individual level. Because the creative arts can enact widespread social action around a topic, ${ }^{28}$ our exhibit has potential for both individual and societal impact.

Using the creative arts to convey women's health information and study its impact is highly innovative. In 2003, Gould ${ }^{31}$ highlighted the historically complex relationship between art and 
science and dispelled the myth that they cannot be used in tandem to pursue and disseminate knowledge. Since that time, the creative arts have been used to convey scientific concepts from the basic sciences, robotics, and other fields ${ }^{44}$ However, the creative arts have rarely been used to convey women's health concepts. For example, in a 2011 scoping review of 71 articles focused on using the creative arts in health research, only $3 \%$ focused on women's health issues and none (0\%) focused on menopause or hot flashes. ${ }^{27}$ In addition, less than $25 \%$ of the 71 articles measured impact on outcomes. ${ }^{27}$ Our exhibit is innovative in addressing these gaps. In summary, the exhibit has high potential to be a disruptive innovation to address the preponderance of myths, misinformation, and negative imagery surrounding menopausal hot flashes and possibly decrease use of unproven therapies. The exhibit conveys a wealth of scientific information and contains balanced and appropriate symbolism. It was thoughtfully and carefully developed and refined with input from scientists, artists, and the general public. Next steps will be to make a business case for full-scale development of the exhibit and showing it locally, nationally, and beyond.

\section{REFERENCES}

1. North American Menopause Society. Menopause Practice: A Clinician's Guide, 4th Edition. Mayfield Heights, OH: North American Menopause Society, 2010.

2. Islam RM, Bell RJ, Billah B, Hossain MB, Davis SR. Prevalence and severity of vasomotor symptoms and joint pain in women at midlife in Bangladesh: a population-based survey. Menopause 2016;23:731-739.

3. Islam RM, Bell RJ, Rizvi F, Davis SR. Vasomotor symptoms in women in Asia appear comparable with women in Western countries: a systematic review. Menopause 2017;24:13131322. 
4. Minkin MJ, Reiter S, Maamari R. Prevalence of postmenopausal symptoms in North America and Europe. Menopause 2015;22:1231-1238.

5. Sriprasert I, Pantasri T, Piyamongkol W, et al. An International Menopause Society study of vasomotor symptoms in Bangkok and Chiang Mai, Thailand. Climacteric 2017;20:171-177.

6. Dennerstein L, Lehert P, Burger HG, Guthrie JR. New findings from non-linear longitudinal modeling of menopausal hormone changes. Hum Reprod Update 2007;13:551-557. 7. Carpenter JS, Groves D, Chen CX, Otte JL, Miller WR. Menopause and big data: word adjacency graph modeling of menopause-related ChaCha data. Menopause 2017;24:783-788. 8. Cumming GP, Currie H, Morris E, Moncur R, Lee AJ. The need to do better - are we still letting our patients down and at what cost? Post Reprod Health 2015;21:56-62.

9. No authors listed. Nonhormonal management of menopause-associated vasomotor symptoms: 2015 position statement of The North American Menopause Society. Menopause 2015;22:1155-1172.

10. No authors listed. The 2017 hormone therapy position statement of The North American Menopause Society. Menopause 2017;24:728-753.

11. Sayakhot P, Vincent A, Teede H. Breast cancer and menopause: partners' perceptions and personal experiences--a pilot study. Menopause 2012;19:916-923.

12. Charbonneau DH. Readability of menopause web sites: a cross-sectional study. J Women Aging 2012;24:280-291.

13. Morris E, Currie H. Provision of menopause information must improve. Menopause Int 2011;17:73-74.

14. Coney S. The menopause industry: how the medical establishment exploits women, First USA Edition. Alameda, CA: Hunter House, 1994. 
15. Kim MK, Seo SK, Chae HD, et al. Perceptions of postmenopausal symptoms and treatment options among middle-aged Korean women. Yonsei Med J 2017;58:533-539.

16. North American Menopause Society. The Menopause Guidebook, Eighth Edition. Mayfield Heights, OH: North American Menopause Society, 2015.

17. Utian WH. Change your menopause! Why one size does not fit all: a doctor's authoritative guide to menopause, healthy aging, and the path to enhanced quality of life for today's women, First Edition. Beachwood, OH: Utian Press, 2011.

18. Duffy O, Iversen L, Hannaford PC. The menopause 'It's somewhere between a taboo and a joke': a focus group study. Climacteric 2011;14:497-505.

19. Pinkerton JV, Zion AS. Vasomotor symptoms in menopause: where we've been and where we're going. $J$ Womens Health (Larchmt) 2006;15:135-145.

20. Donati S, Satolli R, Colombo C, et al. Informing women on menopause and hormone therapy: Know The Menopause a multidisciplinary project involving Local Healthcare system. PloS one 2013;8:e85121.

21. Depypere H, Pintiaux A, Desreux J, et al. Coping with menopausal symptoms: an internet survey of Belgian postmenopausal women. Maturitas 2016;90:24-30.

22. Ma J, Drieling R, Stafford RS. U.S. women desire greater professional guidance on hormone and alternative therapies for menopause symptom management. Menopause 2006;13:506-516.

23. Obermeyer CM, Reynolds RF, Price K, Abraham A. Therapeutic decisions for menopause: results of the DAMES project in central Massachusetts. Menopause 2004;11:456465. 
24. Szpak R, Folwarczny W, Drozdzol A, Szuscik A, Skrzypulec A, Skrzypulec V. [Partner relationships in menopausal period]. Ginekologia polska 2010;81:115-119.

25. Peng W, Adams J, Hickman L, Sibbritt DW. Longitudinal analysis of associations between women's consultations with complementary and alternative medicine practitioners/use of self-prescribed complementary and alternative medicine and menopause-related symptoms, 2007-2010. Menopause 2016;23:74-80.

26. Johnson PJ, Jou J, Rhee TG, Rockwood TH, Upchurch DM. Complementary health approaches for health and wellness in midlife and older US adults. Maturitas 2016;89:36-42.

27. Boydell KM, Gladstone BM, Volpe T, Allemang B, Stasiulis E. The production and dissemination of knowledge: a scoping review of arts-based health research. FQS Forum: Qualitative Social Research 2012;13:32-62.

28. Gergen MM, Gergen KJ. Performative social science and psychology. Historical Social Research 2011;36:291-299.

29. Ede S. Art and Science. London: I. B. Tauris, 2005.

30. Hubard O. Art Museum Education: Facilitating Gallery Experiences. New York: Palgrave MacMillan, 2015.

31. Gould SJ. The Hedgehog, the Fox, and the Magister's Pox: Mending the Gap Between Science and the Humanities, First Edition. New York: Harmony Books, 2003.

32. Lapum J, Church K, Yau T, David AM, Ruttonsha P. Arts-informed research dissemination: patients' perioperative experiences of open-heart surgery. Heart Lung 2012;41:e414. 
33. Lapum J, Ruttonsha P, Church K, Yau T, David AM. Employing the arts in research as an analytical tool and dissemination method: interpreting experience through the aesthetic. Qualitative Inquiry 2012;18:100-115.

34. Lapum JL, Liu L, Church K, et al. Knowledge translation capacity of arts-informed dissemination: a narrative study. Art Research International 2016;1:258-282.

35. Lapum JL, Liu L, Church K, et al. Arts-informed research dissemination in the health sciences: an evaluation of peoples' responses to "The 7,024th Patient" art installation. SAGE Open 2014;4:2158244014524211.

36. Lapum JL, Yau T, Church K. Arts-based research: patient experiences of discharge. British Journal of Cardiac Nursing 2015;10:80-84.

37. Sievert LL. Menopause : a biocultural perspective. New Brunswick, N.J.: Rutgers University Press, 2006.

38. Sievert LL. Variation in sweating patterns: implications for studies of hot flashes through skin conductance. Menopause 2007;14:742-751.

39. Sievert LL, Begum K, Sharmeen T, Chowdhury O, Muttukrishna S, Bentley G. Patterns of occurrence and concordance between subjective and objective hot flashes among Muslim and Hindu women in Sylhet, Bangladesh. Am J Hum Biol 2008;20:598-604.

40. Menopause the Musical(R): A hilarious celebration of women and the change! Available at: http://www.menopausethemusical.com/. Accessed.

41. Bahri N, Yoshany N, Morowatisharifabad MA, Noghabi AD, Sajjadi M. The effects of menopausal health training for spouses on women's quality of life during menopause transitional period. Menopause 2016;23:183-188. 
42. Kiatpongsan S, Carlson K, Feibelmann S, Sepucha K. Decision aid reduces misperceptions about hormone therapy: a randomized controlled trial. Menopause 2014;21:3338.

43. Menard P, Stacey D, Legare F, Woodend K. Evaluation of a natural health product decision aid: a tool for middle aged women considering menopausal symptom relief. Maturitas 2010;65:366-371.

44. Wilson S. Art and Science Now, First Edition. New York, NY: Thames \& Hudson, 2010. 45. Jamin C, Leutenegger E, Senoussi S, Devaux C. [Age fifty and menopause. Complaints expressed and unexpressed by women. Practitioners' perception and therapeutic impact]. Gynecol Obstet Fertil 2005;33:119-125.

46. Guthrie JR, Dennerstein L, Taffe JR, Lehert P, Burger HG. Hot flushes during the menopause transition: A longitudinal study in Australian-born women. Menopause 2005;12:460467.

47. Grisso JA, Freeman EW, Maurin E, Garcia-Espana B, Berlin JA. Racial differences in menopause information and the experience of hot flashes. J Gen Intern Med 1999;14:98-103. 48. Goonaratna C, Fonseka P, Wijeywardene K. Perimenopausal symptoms in Sri Lankan women. Ceylon Med J 1999;44:63-69.

49. Whitehead MI, Whitcroft SIJ, Hillard TC. An atlas of the menopause. Carnforth, Lancs, UK Pearl River, N.Y., USA: Parthenon Pub. Group, 1993.

50. de Salis I, Owen-Smith A, Donovan JL, Lawlor DA. Experiencing menopause in the UK: The interrelated narratives of normality, distress, and transformation. J Women Aging 2018;30:520-540. 
Table 1: Titles, Descriptions, Symbolism, and Sample Information Sources for Seven Art Pieces Title and Description of Exhibit Pieces $\underline{\text { Symbolism }}$ $\underline{\text { Source }}$

1. Interior walls: There are graphic illustrations of About one fourth of women do $45-48$ flames that are about two feet high on three not experience menopausal hot fourths of the interior walls. The blank portion flashes. Hot flashes are a global of the wall contains a sculpture composed of issue. different words and phrases for hot flashes (e.g., hot flush, les bouffées de chaleur).

2. Hot flashes in other cultures: There are three Warm colors (red, orange) dress form mannequins painted with different symbolize the heat of hot skin tones and decorated with warm toned silk flashes. Flowers show hot flowers. The flowers show different areas of flashes as a natural the body where women in different cultures phenomenon. Flowers feel hot flashes.

symbolize blooming and blossoming at menopause.

3. Thermography: There is a short film showing Hot flashes are real, measurable hot flashes captured on a thermal camera. One events. They are not imaginary. woman's image changes colors before, during and after her hot flash. Her companion's image does not change because she does not have a hot flash.

4. Information overload: There is a display case Each stack represents the PubMed containing dolls dressed in period clothing and number of articles listed in the search 
wearing banners for the decades of the 1940's through the 2010's. They stand next to stacks of papers. The stacks range from ankle height (1940’s) to 17 feet high (2010’s).

5. Taking steps to manage hot flashes: There is a floor mat mapping different pathways to different evidence-based hot flash treatments. It is similar to a decision tree or algorithm with questions and arrows leading to additional questions based on previous answers. The floor mat begins with a red arrow labeled start and ends with different types of chairs on a platform that women can sit on.

6. Herbals industry: There is a clear mannequin filled with colorful pills. She stands next to a factory labeled "Herbs, Inc." that is hanging in mid-air. The factory is spilling pills down into a trashcan. Pills and paper money fill the trashcan.
PubMed search engine for that engine decade (yellow) and cumulative graphic for all years including that decade (grey). Information for today's women is literally and figuratively over their heads. Women can take steps literally $\quad 9,10$ and figuratively to find which evidence-based treatments may be best for them. Treatments are on the ground because they are grounded in evidence. The chairs are different to represent the different types of treatments that are available.

The herbals factory hangs in 9 mid-air because use of herbals is not grounded in evidence. Women spend millions of dollars on unproven treatments each year. It symbolizes the medicalization of menopause. 
7. Transformation: There is a glass sculpture of a Flames and heat can be woman rising from a mandala created from transformative - heat causes small silica beads. There are benches nearby. silica to become glass. The benches encourage rest and reflection on the exhibit and its messages.

Source $=$ example scientific informational source that supports accurate information contained in the exhibit art piece.

(C) 2018 The Trustees of Indiana University 
Figure 1: Concept Graphic of Exhibit Piece Titled "Hot Flashes in Other Cultures"

Legend: This is a conceptual rendering created by Brittany Harvey. Flowers depict where women in different cultures feel hot flashes. Women in Bangladesh feel them on the top of their head.

Women in Mexico feel them on the back of their necks. Women in the United States and Europe feel them from their chest to their faces. (C) 2018 The Trustees of Indiana University

Figure 2: Photo of Full-scale Exhibit Piece Titled "Hot Flashes in Other Cultures"

Legend: This is an actual full-scale version of the exhibit piece created and photographed by

Janet S. Carpenter, Indianapolis, IN. (C) 2018 The Trustees of Indiana University

Figure 3: Exhibit Logo

Legend: This is the exhibit logo created by Bridget Gurtowsky, Gurtowsky Graphics, Indianapolis, IN. The exhibit logo mimics the round exhibit shape, symbolizes femininity, the flaming heat of hot flashes, and openness to new information and experiences. (C) 2018 The Trustees of Indiana University 
Figure 1: Concept Graphic of Exhibit Piece Titled "Hot Flashes in Other Cultures"

Legend: This is a conceptual rendering created by Brittany Harvey. Flowers depict where women in different cultures feel hot flashes. Women in Bangladesh feel them on the top of their head.

Women in Mexico feel them on the back of their necks. Women in the United States and Europe feel them from their chest to their faces. (C) 2018 The Trustees of Indiana University

Figure 2: Photo of Full-scale Exhibit Piece Titled "Hot Flashes in Other Cultures"

Legend: This is an actual full-scale version of the exhibit piece created and photographed by

Janet S. Carpenter, Indianapolis, IN. (C) 2018 The Trustees of Indiana University

Figure 3: Exhibit Logo

Legend: This is the exhibit logo created by Bridget Gurtowsky, Gurtowsky Graphics,

Indianapolis, IN. The exhibit logo mimics the round exhibit shape, symbolizes femininity, the flaming heat of hot flashes, and openness to new information and experiences. (C) 2018 The Trustees of Indiana University 